\title{
Simple Design of Wireless Sensor Networks for Traffic Jams Avoidance
}

\author{
Víctor P. Gil Jiménez and M. Julia Fernández-Getino García \\ Department of Signal Theory and Communications, University Carlos III de Madrid, Avenue de la Universidad 30, \\ Leganés, 28911 Madrid, Spain \\ Correspondence should be addressed to Víctor P. Gil Jiménez; vgil@tsc.uc3m.es
}

Received 9 December 2014; Revised 27 March 2015; Accepted 27 March 2015

Academic Editor: Luisa Torsi

Copyright (C) 2015 V. P. Gil Jiménez and M. J. Fernández-Getino García. This is an open access article distributed under the Creative Commons Attribution License, which permits unrestricted use, distribution, and reproduction in any medium, provided the original work is properly cited.

\begin{abstract}
Intelligent transportation systems (ITS) are usually approached by exhaustive measuring and complex signal processing including medium-high cost hardware deployment. In this paper, a novel design of a wireless sensor network system using magnetometers and microphones for the detection and avoidance of traffic jams is described and analyzed. The system, which can also be used for traffic monitoring and surveillance, is simple, energy efficient, and accurate which allows to be implemented with a reduced hardware cost. In order to reduce the maintenance tasks, mini solar panels would also be installed for powering up the motes in the near future.
\end{abstract}

\section{Introduction}

Smart cities are one of the hot topics nowadays. One of the most relevant aspects in a city with this consideration, especially in a big city, is the traffic management and surveillance. In [1], it is shown that the total cost of congestion for the 85 U.S. urban areas is estimated as 65 billion dollars per year, with 3.5 billion hours of delay for the citizens (with the consequent unhappiness) and 5.7 billion gallons of excess in fuel consumption (as well as the excess of pollution generated). For dealing with this problem, traffic surveillance systems are being developed in many countries and a new concept of intelligent transportation systems (ITS) has appeared.

The common approach to tackle the traffic surveillance is an exhaustive monitoring of vehicles [2-4] including counting them, the estimation of the speed [5], the weight, the type of vehicle, and even the number of people inside them [6]. For these purposes, most conventional traffic surveillance systems use intrusive sensors that need to be inserted somehow into the road for monitoring. This is expensive and usually complicated and the life time is shortened because they are most of the times in close contact with vehicles (as well as the number of possible damage cases). In this group we can find inductive loop detectors [7, 8], piezoelectric [9] and other weight or motion detectors. This group of sensors exhibits a very high accuracy (larger than $97 \%$ of detection).

Recently, in the last decade, nonintrusive sensors are being used because of their flexibility and their low-medium cost. Examples are acoustic [10-12], ultrasonic [13], magnetic [14], electromagnetic [15], infrared [16], and even video analysis systems [17-20]. Most of these techniques rely on a wireless sensor network (WSN) [21] or can be easily adapted to be used in a WSN approach. The growth in the development of systems based on WSN in the last decade has increased very quickly due to the huge advantages of this kind of systems. The proposal in this paper also utilizes a WSN, but the simplest and lowest cost one.

There are also other kinds of systems that need some extra hardware at the vehicles such as the global position systems (GPS), mobile-based systems [22], or automatic vehicle identification (AVI) systems. Or there are also other systems based on the cooperation of a large number of users indicating their position and speed (relying on also GPS) to get the global monitoring like in WAZE $[23,24]$ and the like. Since we are looking for a general system valid for all vehicles and independent of how many users use it, these proposals are not considered. 
Focusing now on the application of ITS, users usually only need to know a coarse estimation of the traffic, for example, four-level information: no traffic, low density, medium density, and high density or traffic jam. In these cases, most of the systems already proposed are too much complex and expensive because they are aware of vehicle detection, counting, and the estimation of other parameters that are not used in here. In order to avoid crowded roads or congestions, with these simple 4 levels of information would be enough to be able to make a correct decision on which road to take and thus obtain the final goal of congestions' avoidance. If more levels of information were required, the system could be straightforwardly adapted. The key idea in this paper is to provide a simple yet efficient WSN-based system for giving useful real-time information to prevent congestions and reduce delays.

The rest of the paper is organized as follows. In Section 2, the description of the problem and the scenario is shown. Then, in Section 3, the prototype proposal is presented, analyzed, and discussed. Finally, in Section 4, the main conclusions of this work and future ideas are drawn.

\section{Scenario and Problem Description}

In this section, the main scenario will be described. Besides, the main parameters and technical considerations will also be covered.

As it has been mentioned above, a common scenario is when users have a couple of alternative routes for arriving to the destination. For example, one person might have from two to five different paths for traveling from home to the job, and the decision on which one is the best will depend on the traffic conditions. However, most of the times, the decision has to be made without knowledge of the traffic and once it has been chosen there is no way to return. Moreover, the chosen path can actually be changed or adapted according to the estimation of traffic conditions. If we have extra information about how much traffic there is in the near future it will be very helpful in taking the decision. For example, the decision on whether taking one exit or another will depend on how much traffic there is at the roundabout that follows the exit, but usually you do not have this information. However, for taking a proper decision, you only need to know roughly the amount of vehicles but not their exact number.

In these scenarios, a simple vehicles' detection system is enough. Moreover, the speed or other parameters that are more complicated to be estimated are not required, and, thus, a simpler design is enough for our purposes which impacts the cost yet obtains similar results to other much more complicated schemes.

In Figure 1, a typical scenario is depicted where there is a road where the length of the queue of vehicles, that is, traffic density, is estimated by using four sensors and thus a four-level measurement is obtained. The access point (AP) is also drawn. This AP will receive the information from the last sensor while it is connected with the central station by a mobile network such as general packet radio service (GPRS), universal mobile telecommunications system (UMTS), or long term evolution (LTE). It should be noted

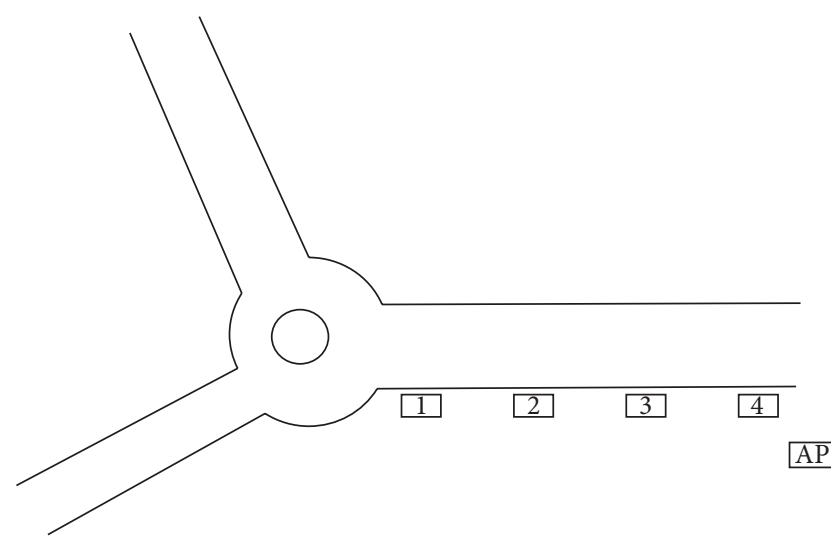

FIGURE 1: Typical scenario with a roundabout where 4 traffic sensors and the access point are placed.

that the system uses a multihop transmission scheme where motes communicate with the motes in their neighbourhood and only the nearer motes to the AP can reach it. In our prototype, this part is implemented through a computer but it could also be integrated into the AP. Although in Figure 1 only one road is monitored, the idea is to deploy this scheme in all the roads where surveillance is required.

The central application will be the sink for all the measurements from every road and will run a server so users can access the information. The user part is a very simple Android application where you can specify the roads that you want to know. The application connects to the server and obtains the results in real-time. If the mobile has the global positioning system (GPS) available, you can also define the position where you want to know the information and when you get to this location, the application will show you the road's state so your decision on next step in your path will be taken with this useful knowledge. Thus, route's decision is taken in a much smarter way and minimizing the traffic jams. If the mobile device does not have GPS or it is not activated, the request must be carried out directly by the user when he/she wants to know the road's state. It should be noted that the server constantly knows the road's state for all the roads being monitored, and, thus, the request from the user only takes a couple of seconds to be ready at the user's terminal.

An example of this scenario is illustrated in Figure 2 where two different alternatives to reach the university are shown. The first road is the shortest one ( 7 minutes, $5.7 \mathrm{~km}$ ), but, however, if there is too much traffic at the roundabout indicated in the figure, the second alternative (much longer: 12 minutes, $10.4 \mathrm{~km}$ ) becomes a better option if there is no traffic there. The key fact is that the choice must be carried out at the point indicated in the figure. Without the knowledge provided by our system, you only can guess which one is the best. However, just a coarse estimation on how much traffic there is at the road falling into the roundabout is enough to make the proper decision. 


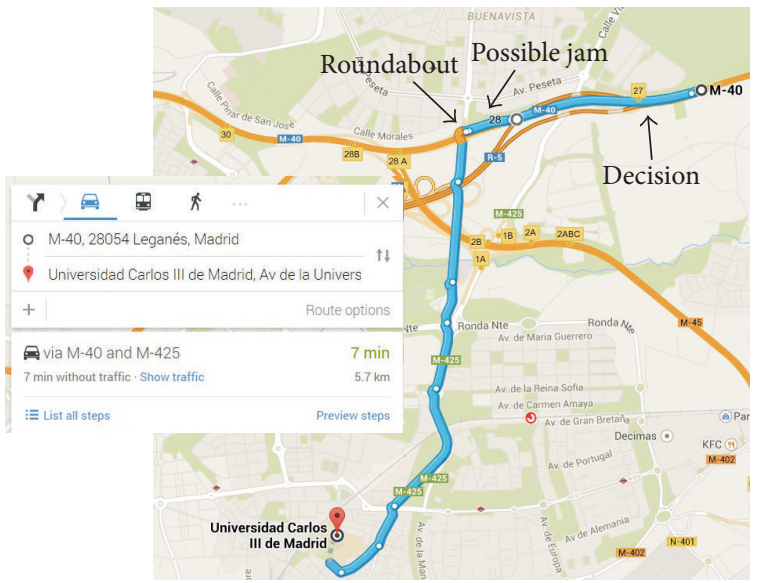

(a) First road

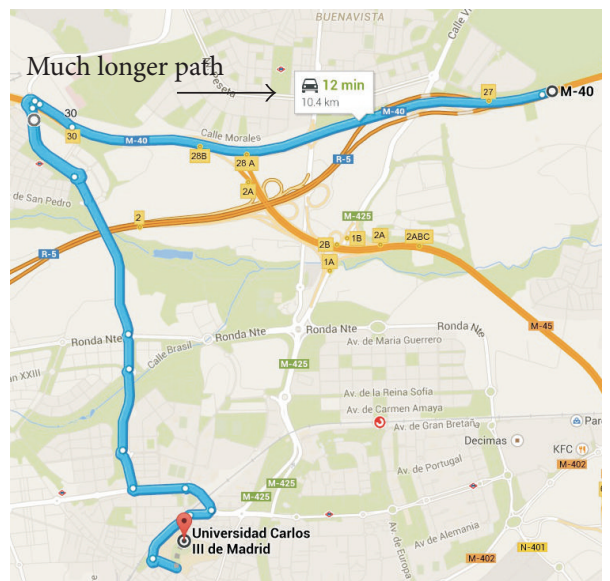

(b) Alternative road

FIgURE 2: Example of two possible roads.

\section{The Prototype}

Once the scenario has been briefly drawn, in this section, the hardware/software scheme proposed for obtaining these coarse estimations, with a simple architecture and design, is described. The architecture is divided into three parts, namely, the wireless sensor network, the central point, and the mobile part. In this paper, the focus is on the wireless sensor network part because the central point is only a piece of hardware/software that receives all the measurements from different access points and runs a server that sends the requested information by the users when it is inquired. The mobile part is a simple Android interface that allows the user to introduce his/her requirements and request the adequate information from the server. Thus, in the following, the paper will describe only the WSN, which is composed of both a hardware part and a software part.

3.1. Hardware. As it has been indicated, a wireless sensor network has been used for the proposal, specifically, the MICAz motes from Crossbow. These very simple motes are composed of three parts, namely, (1) the MICAz OEM reference board where the radio frequency and signal processing modules are allocated, (2) the MTS310 sensor board, where several sensors are available, and (3) the power supply, which in this prototype is AA batteries but in the near future will be a small yet power efficient solar panel to provide enough energy to the mote.

The MICAz OEM reference board allows IEEE 802.15.4/ ZigBee compliant wireless mesh networking nodes in the $2.4 \mathrm{GHz}$ band with up to $250 \mathrm{kbps}$. In our prototype, a physical layer with less than $1 \mathrm{kbps}$ is enough. The board provides a $128 \mathrm{~Kb}$ programmable flash memory where our algorithm and system can be allocated along with a $512 \mathrm{~Kb}$ memory for storing measurements. Actually, our implementation only needs less than $1 \mathrm{~Kb}$ of storage. The board also has 8 channels analog to digital converter (ADC) of 10-bit resolution for acquiring data from the different sensors. The maximum transmit power is $3 \mathrm{dBm}$ and the sensibility is $-94 \mathrm{dBm}$.

Connected to this reference board, there is a MTS310 sensor board. This MTS10 provides different sensors such as a microphone, a sounder, a light sensor based on a simple CdSe photocell, a thermistor from Panasonic (ERT-J1VR103J) for temperatures in the range from $-40^{\circ}$ to $70^{\circ}$, a 2-axis accelerometer from Analog Devices (ADXL202JE) with a range of $\pm 2 \mathrm{~g}$, a resolution of $2 \mathrm{mG}$ and a sensitivity of $167 \mathrm{mV} / \mathrm{G} \pm 17 \%$, and, finally, the 2-axis magnetometer from Honeywell (HMC1002), with a sensitivity of $3.2 \mathrm{mV} / \mathrm{V} /$ gauss and a resolution of $27 \mu$ gauss. This highly sensitive $\mathrm{NiFe}$ coating causes a bridge resistance of the circuit to change. This bridge is very sensitive and is able to measure the earth's field and other small magnetic fields. Since the vehicles have a large ferrous mass, when vehicles are close to the sensor, they distort the earth's magnetic field and these distortions can be measured by the sensor [14]. The differences between our approach and others in the literature like [14] are significant. First, in our approach, the motes are on the side of the road, typically at the railing bars, that are disposed to prevent vehicles going outside the road, instead of being inside or on the road. This scheme is better than previous proposals because it is cheaper in terms of installation and maintenance, although it is more challenging from the point of view of the detection. However, for our purposes, the important estimation is the presence or absence of either a stopped or low motion vehicle in the surroundings of the sensor which would indicate that the traffic is crowded at this point. This is indeed a second difference because other proposals try to estimate and/or count the vehicles while moving (usually much more complicated). The third difference is that our proposal provides enough useful information as others in the literature with much less complexity. The range of detection of this scheme is between 0.45 and 2 meters, which is usually enough. In order to reduce the estimation errors, also the microphone is used to record 1 second of sound. With a simple signal processing of both signals, the presence or absence of either a stopped or low speed vehicle near 


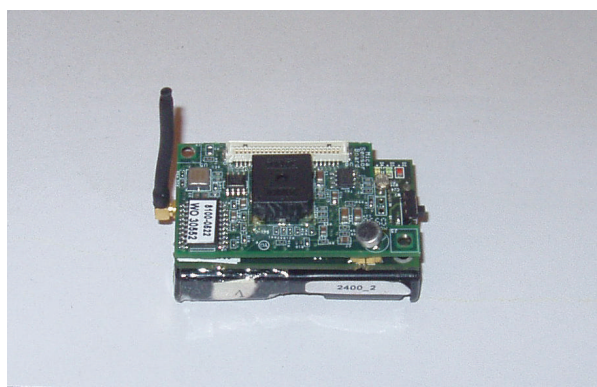

(a) MICAz mote

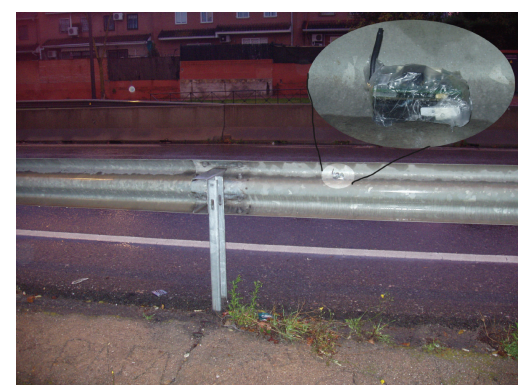

(b) Installed MICAz motes

Figure 3: The MICAz motes for vehicles' detection.

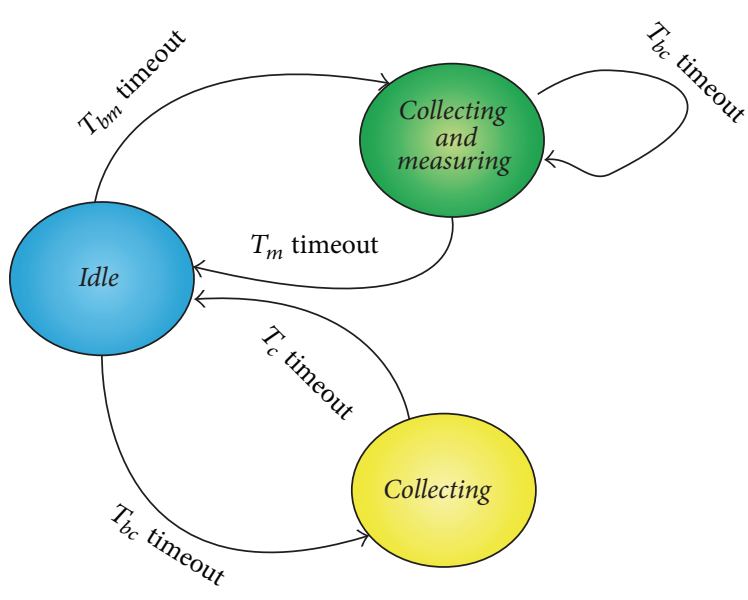

FIgURE 4: State diagram at the motes.

the sensor can be estimated with reliability larger than $80 \%$. Besides, the detection range can be extended to 3 meters.

Finally, the 2-axis accelerometer is used to detect if the motes are being stolen. Since these systems are deployed through the roads without supervision, although they will be hidden, one could realize their presence and try to steal them. In this case, the mote detects the movement and activates an alarm. At the central point, the server could establish a mechanism for informing about this issue and the timing reference. With the traffic cameras and the time and direction obtained from the 2-axis accelerometer the thief could be located.

In Figure 3, the low cost mote used in this proposal is shown and also an installed one. As it can be observed, they have been protected by a plastic bag to save them from the rain and dust.

3.2. Software or Signal Processing. The other important part of the scheme is the software and the signal processing associated. The software at the motes has been programmed using the TinyOS, and the state diagram can be seen in Figure 4. There are three states, namely, Idle, Collecting, and Collecting and measuring. The description of which tasks are carried out on each state is provided in the following:

(i) Idle. In this state the mote is in sleep mode. (ii) Collecting. In this state, the mote awakes and stays listening to messages from other motes during a time of $T_{c}$ (collecting time). If it receives a message from the previous adjacent mote, it forwards it to the next one. After a time of $T_{c}$, it moves to the idle state. It should be pointed out here that the first motes cannot reach directly the access point, and, so, a multihop communication scheme is used. In this sense, the number of motes indicates the order and thus the previous adjacent mote for mote 3 is mote number 2 and so on. In order to increase reliability and robustness and also to prevent retransmissions due to collisions or propagation conditions, the design provides redundancy in the following sense. The motes can reach not only the precedent and next mote but also some others. In this way, motes can receive data from other ones, which reduces the failures in the reception and also the retransmissions because it is less likely that the message from one mote was not heard by at least another one. This configuration also provides robustness against malfunctioning of one mote (or even if this mote is damaged or stolen) since the messages can still be received by the other motes and, hence, forwarded.

(iii) Collecting and Measuring. In this state, the mote awakes for a period of $T_{m}$ (measuring time) and, during this time, it stays listening to other motes' messages but also it performs two measurements, one at the beginning of $T_{c}$ and the other one at the end. After that, it sends a message to the next mote with its two measurements and jointly the others' motes measurements, extracted from the messages received from other motes. After $T_{m}$, it goes back to idle state again. For this state, the explanations from previous state hold too. The purpose of the two measurements is to avoid erroneous measurements and, thus, false positives or negatives.

The normal sequence for a mote is the following. The mote starts in idle state, and, after $T_{b m}$ (time between measurements), the mote goes to collecting and measuring state during $T_{m}$. After this time, the mote goes to idle state for $T_{b c}-T_{m}$ time, with $T_{b c}$ being the time between two intervals when the motes collect messages from other ones. After this 


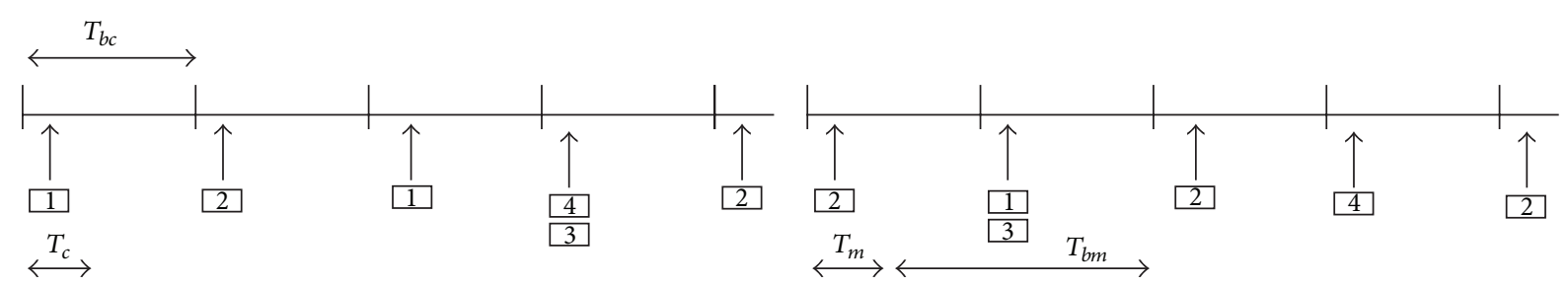

(a)

(b)

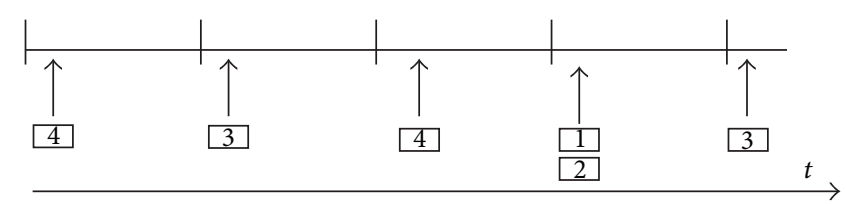

(c)

Figure 5: Timing schedule for scenario in Figure 1 for three different situations: (a) low traffic load, (b) medium-low traffic load, and (c) high traffic load or traffic jam.

time, the mote jumps to the collecting state for a time of $T_{c}$, and, after that, it goes back again to idle state for a period of $T_{b c}-T_{c}$. This sequence is always the same but $T_{b m}$ can vary during the operation. Basically, it depends on how much traffic there is and the purpose is to maintain the most active mote close to the position of the end of the traffic queue. The idea is simple, if there are either stopped or low motion vehicles in the surrounding of mote 3 in Figure 1, mote 3 is the one that should be most active measuring to detect if the traffic increases or decreases, because for sure mote number 1 in the next seconds will measure traffic because the queue reaches mote 3 . Thus, the $T_{b m}$ is changed dynamically and distributively during the operation time based on how much traffic there is in the road being monitored (each mote changes their $T_{b m}$ according to the messages they receive from other motes with the estimation of traffic in order to guarantee that the mote closer to the end of the traffic queue is the most active one).

In Figure 5, three different scenarios are depicted: (a) when there is low traffic or no traffic at all, (b) when there is medium-low traffic, and (c) when the road is crowded and, therefore, vehicles reach mote 4 . In Figure 5, the number in the square indicates which mote is measuring. In Summary of Time Parameters section, all the different times are shown. The following relationships must be fulfilled:

$$
\begin{aligned}
T_{b m} & \geq T_{c}, \\
T_{b m} & \geq T_{m}, \\
T_{m} & \geq 3 \mathrm{sec}, \\
T_{c} & \geq T_{m}
\end{aligned}
$$

although, usually, $T_{c}=T_{m}$. The updating time $\left(T_{u}\right)$ is $N_{s} \times$ $T_{b m}$, where $N_{s}$ is the number of sensor motes. By using these expressions we can establish the different times based on the updating time required. For a $T_{u}=1$ minute (a reasonable value) and $N_{s}=4$, the $T_{b m}$ is 25 seconds, which makes the system very energy efficient.
In the collecting and measuring state, the measuring process involves four measurements, two from the 2-axis magnetometer and two from the microphone. At the beginning of measuring time, the sensor wakes up and takes the value from the 2-axis magnetometer, and, at the same time, records 1 second of sound from the microphone. Then, 1.5 seconds before the $T_{m}$ expires, it takes a second measurement from the 2-axis magnetometer and another 1 second of sound from microphone. The reason of taking two measurements at two instants is because our purpose is to detect if there is either a stopped or low motion vehicle close to the mote or not and, therefore, in order to avoid temporary detections, two measurements are acquired.

The analysis of measurements at the motes is very simple. The magnetometer's measurements are compared to a threshold estimated during the calibration of the system. This simple comparison is enough to estimate the presence or absence of a vehicle close to the mote. It should be noted here that this calibration procedure is simple and can be carried out before deployment.

As mentioned before, in order to reduce errors, an auxiliary measurement from a microphone is also acquired. The idea is to register the sounds in the surroundings of the mote to evaluate whether it is likely that a stopped vehicle is present or not. The signal analysis is also very simple. If the recorded signal is $s[n]$, the processed signal is

$$
p[k]=\frac{1}{E[s[n]]} \sum_{n=0}^{N-1} s[n] e^{-j(2 \pi n k / N)},
$$

where $E[s[n]]$ is the energy of the signal $s[n]$ and it is used to reduce the environmental components. The equation above is the fast Fourier transform (FFT) calculation. If there are important components below $200 \mathrm{~Hz}$, it is very likely that a vehicle is close to the mote; if there are also important components up to $600 \mathrm{~Hz}$, it is very likely that a stopped vehicle would be close to the mote. Above $900 \mathrm{~Hz}$, the sources of these components are different from the engine, like music or klaxon. If there is no vehicle close to the mote, 


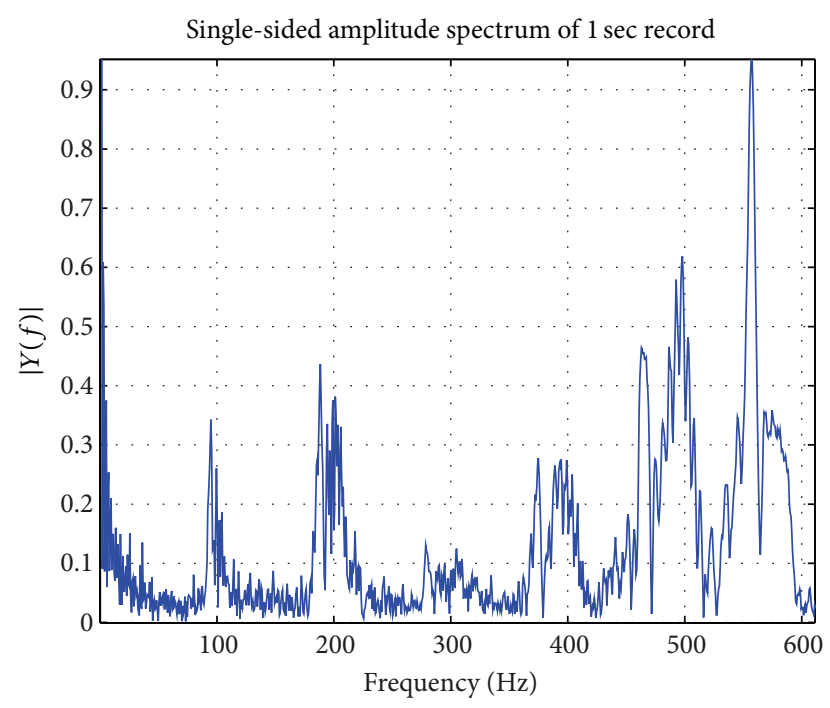

(a) Slow motion vehicle

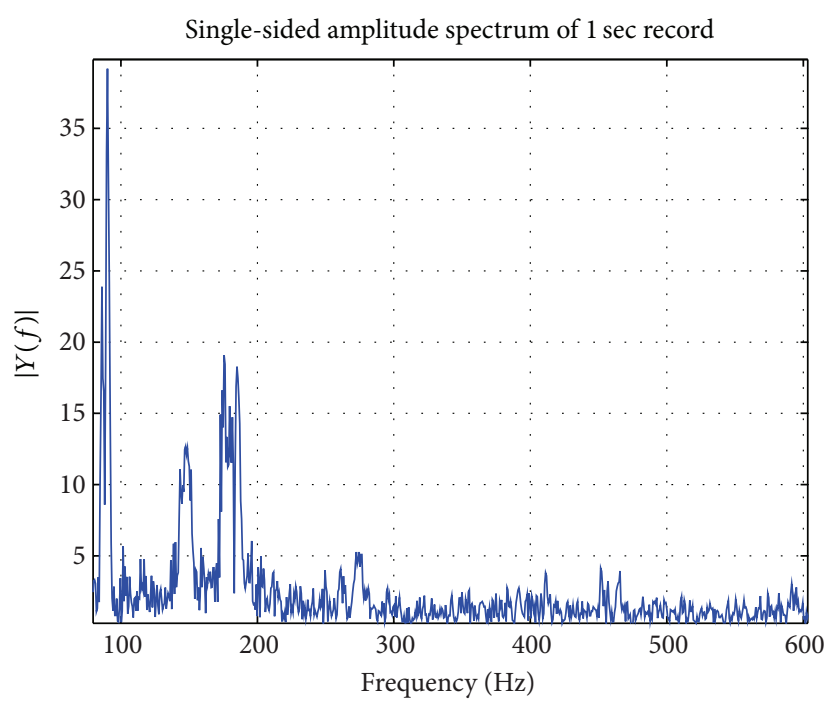

(b) Moving vehicle

FIGURE 6: Spectrum of a recorded 1 second of sounds from vehicles.

no components at all will be found. So, since our goal is to estimate the presence of stopped or low speed vehicles, we are interested in components below $600 \mathrm{~Hz}$. Two examples of recorded signals can be seen in Figure 6. Besides, the mean energy of the sound is usually higher for moving vehicles than stopped ones, and, so, $E[s[n]]$ is another important parameter. Again, if this parameter is too high it is due to other noise sources like klaxon or music.

The data to be sent in a message will be the information received from the previous adjacent mote (this length will be different if the mote is number 2 or if it is number 3 ) and the information measured in the current mote: one bit per measurement from the magnetometer indicating if there is a vehicle or not and then one bit per measurement from the microphone indicating if there is a vehicle or not. This message is protected by using a parity check bit per measurement. Besides, each hour, the battery level is also sent once by the motes in their messages. In this way, the AP has an estimation about the battery level from all the motes. At the AP, all the measurements are received and processed to evaluate what the traffic level is. Some incoherent measurements or false positives or negatives can be solved. For example, if motes 1, 3, and 4 estimate that there is a stopped vehicle but mote 2 does not, it is easy to find that the estimation from mote 2 was erroneous, because it is highly likely that the queue reaches mote number 4 and, thus, also mote number 2 .

The same principle holds for other situations or scenarios. For example, a vehicle crashes near the mote but the engine still runs although the vehicle is stopped and is outside the road. In this case, it does not interfere with the traffic and other vehicles can continue their trip. In this scenario, even though the mote will indicate that there is traffic jam until this point, since the other ones will not, this situation will be treated by the postprocessing as false positive and it will be automatically corrected and the given answer will be that there is no traffic jam, which is the correct one, indeed.

All the information from the AP is transmitted to the server to provide the service to the end users.

Although this system is focused on intersections or roundabout-like scenarios, if there is a congested long road, for sure it will collapse one or more closely placed roundabouts or intersections too, where our system can be installed, and, hence, those scenarios are also covered by this proposal, saving a lot of resources and obtaining similar results as other more complex systems.

\section{Conclusions and Future Work}

The ITS is a hot topic nowadays in big cities and in particular when defining the future smart cities. There is large literature on proposals for dealing with this problem. However, in most of the cases, the proposals are too much complex and sophisticated providing much more information than required. In this paper, a simple yet low cost and energy efficient scheme is proposed to tackle the problem of avoidance of traffic jams. The proposed scheme is simple and only needs low cost hardware to be implemented. It is also energy efficient since the number of transmissions and the amount of information bits are significantly low, and the system has proven to work properly in a small-scale scenario. The future work is the deployment of a large-scale experiment with more roads being analyzed and a more powerful software at the server. Also, in order to reduce the battery drain, mini solar panels will be installed into each mote to supply the energy. In this case, the system maintenance is very low. Besides, a more sophisticated and complex mobile application could be designed based on these coarse estimations of the amount of vehicles. 


\section{Summary of Time Parameters}

$T_{b c}$ : Time between collecting messages-this is the time between two periods of collecting messages from other adjacent motes

$T_{c}$ : Collecting time-this is the duration of the collecting time

$T_{m}$ : Measuring time-his is the duration of a period of measuring

$T_{b m}$ : Time between measurements-this is the time between two periods of measurements

$T_{u}$ : Updating time-this is the time between two updates in information per mote, that is, this time indicates how fast motes update their information.

\section{Conflict of Interests}

The authors declare that there is no conflict of interests regarding the publication of this paper.

\section{Acknowledgments}

This work has been partially funded by the Spanish National Projects GRE3N-SYST (TEC2011-29006-C03-03) and COMONSENS (CSD2008-00010).

\section{References}

[1] D. Schrank and T. Lomax, "2005 urban mobility report," Tech. Rep., Texas Transportation Institute, The Texas A\& M University System, College Station, Tex, USA, 2005.

[2] X. Laisheng, P. Xiaohong, W. Zhengxia, X. Bing, and H. Pengzhi, "Research on traffic monitoring network and its traffic flow forecast and congestion control model based on wireless sensor network," in Proceedings of the International Conference on Measuring Technology and Mechatronics Automation (ICMTMA '09), vol. 1, pp. 142-147, Hunan, China, April 2009.

[3] J. Zhou, C. L. P. Chen, and L. Chen, "A small-scale traffic monitoring system in urban wireless sensor networks," in Proceedings of the IEEE International Conference on Systems, Man, and Cybernetics (SMC '13), pp. 4929-4934, October 2013.

[4] J. Fernãndez, L. Calavia, C. Baladrón et al., "An intelligent surveillance platform for large metropolitan areas with dense sensor deployment," Sensors, vol. 13, no. 6, pp. 7414-7442, 2013.

[5] R. K. Megalingam, V. Mohan, A. Mohanan, P. Leons, and R. Shooja, "Wireless sensor network for vehicle speed monitoring and traffic routing system," in Proceedings of the 2nd International Conference on Mechanical and Electrical Technology (ICMET '10), pp. 631-635, IEEE, Singapore, September 2010.

[6] C. Chen, J. Kwon, J. Rice, A. Skabardonis, and P. Varaiya, "Detecting errors and imputing missing data for single loop surveillance systems," in Proceedings of the Transportation Research Board (TRB) 82nd Annual Meeting, Washington, DC, USA, January 2003.

[7] S. Oh, S. G. Ritchie, and C. Oh, "Real time traffic measurement from single loop inductive signatures," in Proceedings of the Transportation Research Board (TRB) 81st Annual Meeting, Washington, DC, USA, January 2002.
[8] X. Zhang, Y. Wang, and N. L. Nihan, "Monitoring a freeway network in real-time using single-loop detectors: system design and implementation," in Proceedings of the 83rd TRB Annual Meeting, Washington, DC, USA, January 2004.

[9] C. J. Wynant, "Quartz technology for weigh-in-motion sensors," Tech. Rep., Kistler Instruments, 2002.

[10] A. Y. Nooralahiyan, H. R. Kirby, and D. McKeown, "Vehicle classification by acoustic signature," Mathematical and Computer Modelling, vol. 27, no. 9-11, pp. 205-214, 1998.

[11] B. Barbagli, L. Bencini, I. Magrini, G. Manes, and A. Manes, "A real-time traffic monitoring based on wireless sensor network technologies," in Proceedings of the 7th International Wireless Communications and Mobile Computing Conference (IWCMC '11), pp. 820-825, July 2011.

[12] Y. M. Kwon, K. Mechitov, S. Sundresh, W. Kim, and G. Agha, "Resilient localization for sensor networks in outdoor environments," Tech. Rep., 2005.

[13] Y. Jo and I. Jung, "Analysis of vehicle detection with wsn-based ultrasonic sensors," Sensors, vol. 14, no. 8, pp. 14050-14069, 2014.

[14] S. Coleri, S. Y. Cheung, and P. Varaiya, "Sensor networks for monitoring traffic," in Proceedings of the Allerton Conference on Communication, Control and Computing, September 2004.

[15] A. Duzdar and G. Kompa, "Applications using a low-cost baseband pulsed microwave radar sensor," in Proceedings of the 18th IEEE Instrumentation and Measurement Technology Conference, vol. 1, pp. 239-243, IEEE, Budapest, Hungary, May 2001.

[16] Y. Iwasaki, M. Misumi, and T. Nakamiya, "Robust vehicle detection under various environmental conditions using an infrared thermal camera and its application to road traffic flow monitoring," Sensors, vol. 13, no. 6, pp. 7756-7773, 2013.

[17] C. A. MacCarley, S. Hockaday, D. Need, and S. Taff, "Detecting errors and imputing missing data for single loop surveillance systems," in Proceedings of the TRB Annual Meeting, Washington, DC, USA, January 1992.

[18] Al Bovik, S. T. Waller, R. Heath, and S. Vishwanath, "Wireless visual sensor networks for urban traffic management," Tech. Rep., University of Texas at Austin, Austin, Tex, USA, 2010.

[19] J. Leitloff, D. Rosenbaum, F. Kurz, O. Meynberg, and P. Reinartz, "An operational system for estimating road traffic information from aerial images," Remote Sensing, vol. 6, no. 11, pp. 1131511341,2014

[20] J. Guo, J. Wang, X. Guo, C. Yu, and X. Sun, "Preceding vehicle detection and tracking adaptive to illumination variation in night traffic scenes based on relevance analysis," Sensors, vol. 14, no. 8, pp. 15325-15347, 2014.

[21] F. A. Al-Nasser and M. S. Mahmoud, "Wireless sensors network application: a decentralized approach for traffic control and management," in Wireless Sensor Networks: Technology and Applications, chapter 16, pp. 347-374, InTech, 2012.

[22] Y. Zhao, "Mobile phone location determination and its impact on intelligent transportation systems," IEEE Transactions on Intelligent Transportation Systems, vol. 1, no. 1, pp. 55-64, 2000.

[23] B. McClendon, "Google maps and waze, outsmarting traffic together," 2013, http://googleblog.blogspot.com/2013/06/ google-maps-and-waze-outsmarting.html.

[24] WAZE, "Waze official web page," 2015, https://world.waze.com/. 

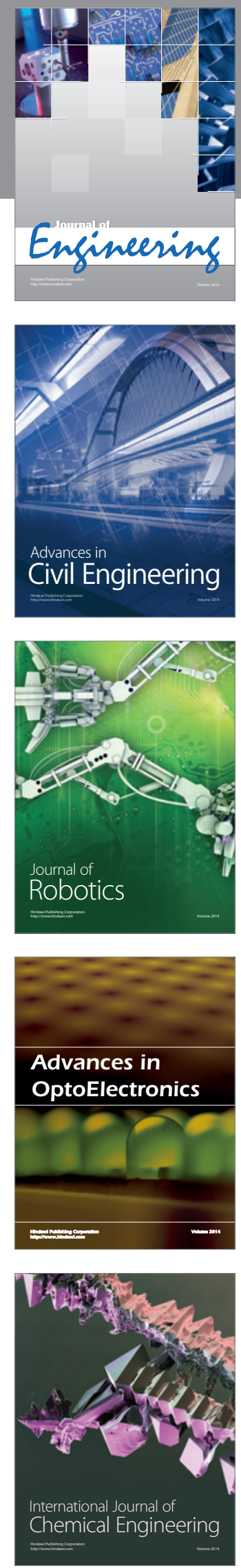

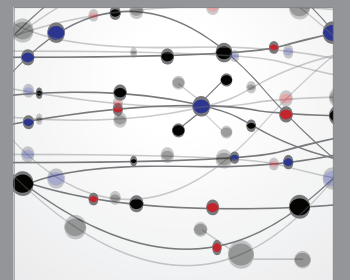

The Scientific World Journal
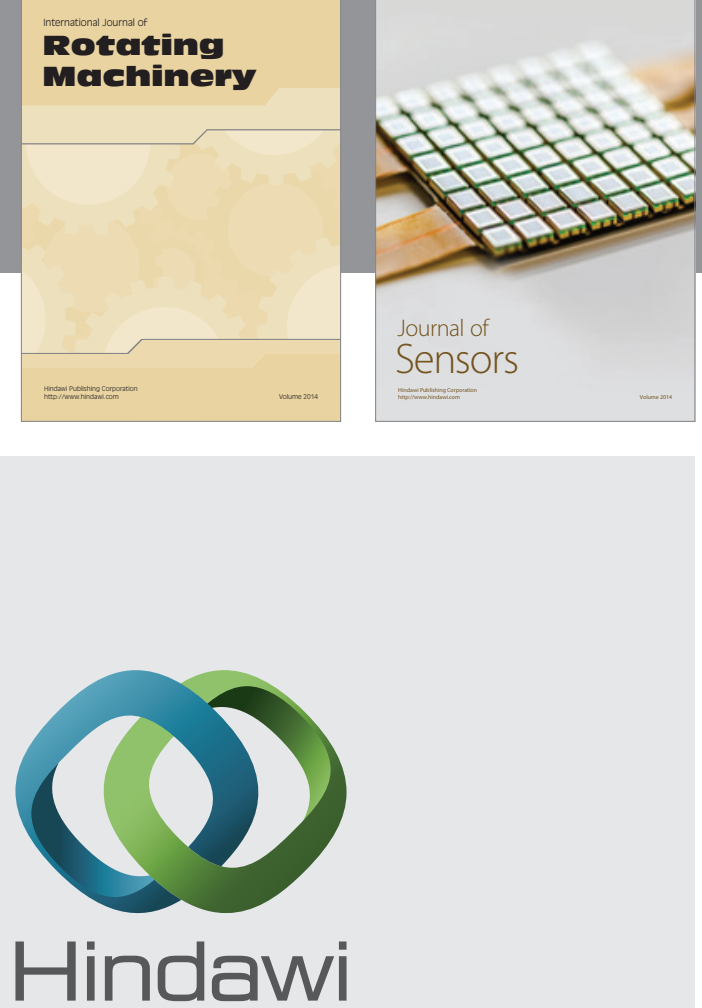

Submit your manuscripts at http://www.hindawi.com
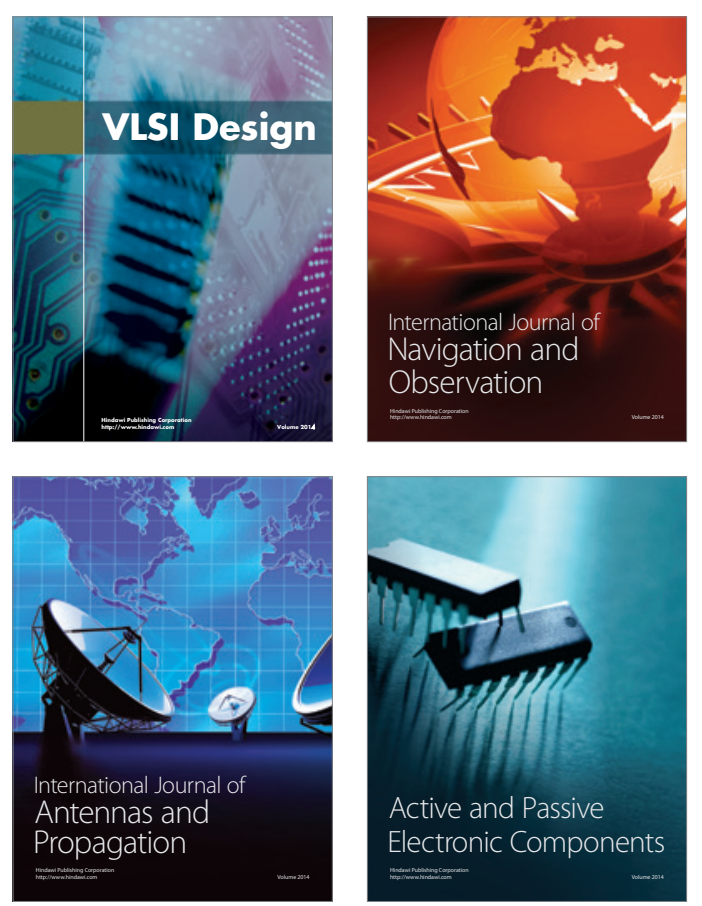
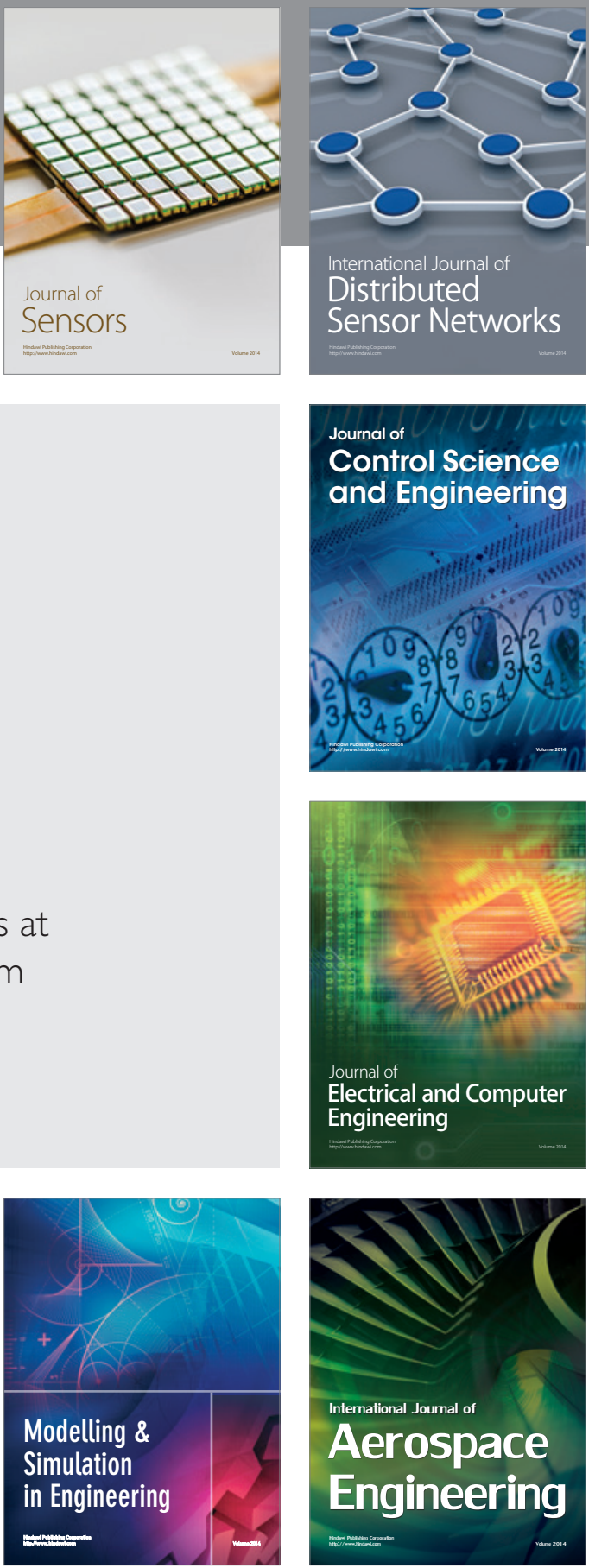

Journal of

Control Science

and Engineering
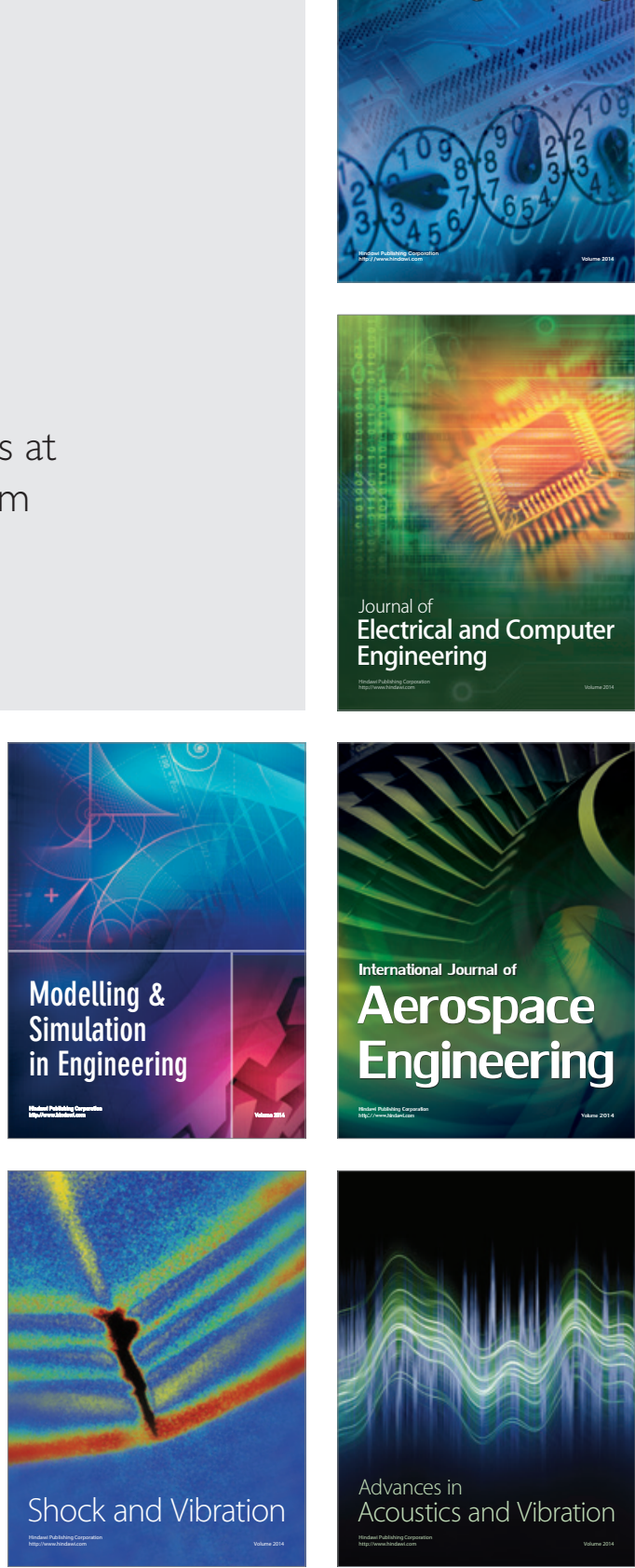